\title{
BMJ Open Comparative assessment of onabotulinumtoxinA and mirabegron for overactive bladder: an indirect treatment comparison
}

\author{
Nick Freemantle, ${ }^{1}$ David A Ginsberg, ${ }^{2}$ Rachael McCool, ${ }^{3}$ Kelly Fleetwood, ${ }^{4}$ \\ Mick Arber, ${ }^{3}$ Kristin Khalaf, ${ }^{5,6}$ Clara Loveman, ${ }^{7}$ Quanhong Ni, ${ }^{8,9}$ Julie Glanville ${ }^{3}$
}

To cite: Freemantle N, Ginsberg DA, McCool R, et al. Comparative assessment of onabotulinumtoxinA and mirabegron for overactive bladder: an indirect treatment comparison. BMJ Open 2016:6:e009122.

doi:10.1136/bmjopen-2015009122

- Prepublication history for this paper is available online. To view these files please visit the journal online (http://dx.doi.org/10.1136/ bmjopen-2015-009122).

Received 17 June 2015 Revised 15 January 2016 Accepted 25 January 2016

\section{CrossMark}

For numbered affiliations see end of article.

\section{Correspondence to}

Professor Nick Freemantle; nicholas.freemantle@ucl. ac.uk

\section{ABSTRACT}

Context: OnabotulinumtoxinA and mirabegron have recently gained marketing authorisation to treat symptoms of overactive bladder (OAB).

Objective: To evaluate the relative efficacy of mirabegron and onabotulinumtoxinA in patients with idiopathic OAB.

Design: Network meta-analysis.

Data sources: A search of 9 electronic databases, review documents, guidelines and websites.

Methods: Randomised trials comparing any licensed dose of onabotulinumtoxinA or mirabegron with each other, anticholinergic drugs or placebo were eligible (19 randomised trials were identified). 1 reviewer extracted data from the studies and a second reviewer checked the data. Candidate trials were assessed for similarity and networks were developed for each outcome. Bayesian network meta-analysis was conducted using both fixed-effects and random-effects models. When there were differences in mean baseline values between mirabegron and onabotulinumtoxinA trials they were adjusted for using network metaregression (NMR).

Results: No studies directly comparing onabotulinumtoxinA to mirabegron were identified. A network was created for each of the 7 outcomes, with 3-9 studies included in each individual network. The trials included in the networks were broadly similar. Patients in the onabotulinumtoxinA trials had more urinary incontinence and urgency episodes at baseline than patients in the mirabegron trials and these differences were adjusted for using NMR. Both onabotulinumtoxinA and mirabegron were more efficacious than placebo at reducing the frequency of urinary incontinence, urgency, urination and nocturia. OnabotulinumtoxinA was more efficacious than mirabegron (50 and $25 \mathrm{mg}$ ) in completely resolving daily episodes of urinary incontinence and urgency and in reducing the frequency of urinary incontinence, urgency and urination. NMR supported the results of the network meta-analysis.

Conclusions: In the absence of head-to-head trials comparing onabotulinumtoxinA to mirabegron, this indirect comparison indicates that onabotulinumtoxinA may be superior to mirabegron in improving symptoms

\section{Strengths and limitations of this study}

- This study represents the first evaluation of the relative benefits of onabotulinumtoxinA compared with mirabegron in adults with idiopathic overactive bladder.

- The results enhance the current evidence base, assisting patients and healthcare providers to make more informed decisions about treatment options.

- In the absence of head-to-head trials, the analyses relied on indirect comparisons, which make underlying assumptions about the similarity of the trials and participants included in the network.

- Network meta-regression was applied to the changes from baseline in urinary incontinence episodes and urgency episodes, in order to adjust for differences in the baseline values of these outcomes. Because individual patient data were available for onabotulinumtoxinA trials, but not for mirabegron trials, it was assumed that the relationship between baseline values and change from baseline was the same across all included trials.

- This analysis did not include an assessment of adverse events.

of urinary incontinence, urgency and urinary frequency in patients with idiopathic OAB.

\section{INTRODUCTION}

Overactive bladder $(\mathrm{OAB})$ is defined by the International Continence Society (ICS) as "urgency, with or without urgency incontinence usually with increased daytime frequency and nocturia." ${ }^{\prime 1}$ The aetiology of $\mathrm{OAB}$ is diverse, but in the majority of instances the underlying cause of $\mathrm{OAB}$ is unclear (idiopathic). 
$\mathrm{OAB}$ and urinary incontinence (UI) are associated with recurrent urinary tract infection and skin breakdown, infection, and/or ulceration. ${ }^{2}{ }^{3}$ UI is also associated with considerable social stigma, may contribute to loss of independence, and significantly reduces health-related quality of life (HRQoL). ${ }^{2}{ }^{4}$ In addition, there seems to be a relationship between symptoms of $\mathrm{OAB}$ and the risk of falls and fractures among elderly patients. $^{5}$

Although there is no cure for $\mathrm{OAB}$, symptoms may be managed through a range of approaches. Treatment generally follows a stepped approach from conservative to more invasive interventions. ${ }^{6-8}$ Treatment pathways initially focus on lifestyle intervention (weight loss, caffeine and alcohol reduction), behavioural techniques (bladder training, bladder control strategies, pelvic floor muscle training and fluid management) and oral pharmacotherapy, predominantly anticholinergics (in some countries, mirabegron is available as an initial option). If these therapies fail then other options include mirabegron, botulinum toxin injection, sacral nerve stimulation, posterior tibial nerve stimulation and surgery. The National Institute for Health and Care Excellence recommends no more than two oral agents for UI. ${ }^{9}$ However, despite the recognition that alternative treatments are available which may be more suitable, patients tend to continue to cycle on oral drugs without further improvement. ${ }^{10}$ The physical and psychological sequelae of inadequately managed $\mathrm{OAB}$ can be debilitating and have a significant impact on patients' overall HRQoL. ${ }^{11} 12$

The most recently approved agents to treat $\mathrm{OAB}$ are onabotulinumtoxinA (BOTOX; Allergan, Inc) and mirabegron (Myrbetriq, Betmiga; Astellas Pharma). ${ }^{13-16} \mathrm{~A}$ recent systematic review and mixed treatment comparison has been published comparing mirabegron $50 \mathrm{mg}$ to antimuscarinics for the management of OAB. ${ }^{17}$ However, to date, there have been no such comparisons that have included onabotulinumtoxinA.

In the absence of head-to-head data, indirect treatment comparisons provide the best available evidence to allow for informed decisions between different treatments. The objective of this study was to evaluate the relative clinical efficacy of the licensed dose of onabotulinumtoxinA (100 U) compared with mirabegron $(25$ and $50 \mathrm{mg}$ ) in adults with $\mathrm{OAB}$ via an indirect treatment comparison.

\section{METHODS}

\section{Eligibility criteria}

The eligibility of studies for inclusion in the network meta-analysis (NMA) was established a priori and documented in a project protocol. Eligible studies were randomised controlled trials, published in English, which compared onabotulinumtoxinA or mirabegron with each other, anticholinergic drugs, or placebo in adults with idiopathic OAB (table 1).

\section{Study identification and selection}

The search strategies for this update review were informed by a previous literature review, ${ }^{18}$ with additional terms identified through a range of approaches, including scanning background literature, browsing database thesauri and use of the online PubMed PubReminer tool (http://hgserver2.amc.nl/cgi-bin/ miner/miner2.cgi). A search of nine databases (from inception to the end of August 2013), relevant reviews and guidelines, and websites was conducted to identify all relevant trials that might contribute to the NMA. Details of the information resources searched and electronic search strategies for MEDLINE and EMBASE, including any limits used, are reported in the online supplementary appendices A1-A2. The search strategies for other data sources are available on request.

Search results were assessed for relevance according to the eligibility criteria, with records screened in several stages (figure 1). One reviewer selected the studies and a second reviewer carried out a quality check on a sample of records. Any differences in decisions were resolved through discussion and/or by consulting a third reviewer. Studies excluded after examination of the full text are listed, along with the reason for exclusion, in online supplementary appendix B1.

\section{Data extraction and study assessment}

An initial network was developed incorporating all the identified trials. This was then adapted for each outcome, following the feasibility assessment, to reflect the trials that actually reported data for that outcome.

NMA requires that the identified studies are suitably homogeneous to facilitate reliable comparison. ${ }^{19}$ Data were extracted from the studies to inform both this similarity assessment and the outcome analyses for the networks developed. One reviewer extracted key study characteristics relating to study methods, populations, trial settings, treatments and outcomes using a standardised data extraction form, and a second reviewer checked each extraction. Any discrepancies were resolved by discussion, or with the input of a third reviewer. The similarity of the candidate studies was assessed using a modified version of the tool developed by the Australian Pharmaceutical Benefits Advisory Committee $(\mathrm{PBAC})^{20}$ (see online supplementary appendix $\mathrm{C} 1$ ).

\section{Heterogeneity assessment}

For each outcome, statistical heterogeneity was assessed for each pairwise treatment comparison informed by at least two trials. Heterogeneity was measured by the $\mathrm{I}^{2}$ statistic, where $\mathrm{I}^{2}$ values of $25 \%, 50 \%$ and $75 \%$ were considered to indicate low, moderate and high heterogeneity, respectively. ${ }^{21}$

\section{Network meta-analysis}

The analysis focused on the licensed doses of onabotulinumtoxinA (100 U) and mirabegron (25 and 
Table 1 Eligibility criteria for network meta-analysis

\begin{tabular}{|c|c|c|}
\hline $\begin{array}{l}\text { PICOS } \\
\text { element }\end{array}$ & Inclusion criteria & Exclusion criteria \\
\hline Population & $\begin{array}{l}\text { Adults ( } \geq 18 \text { years); } \\
\text { Idiopathic overactive bladder, with/without } \\
\text { urinary incontinence; } \\
\text { Conditions described as: } \\
\text { - Refractory detrusor overactivity; } \\
\text { - Idiopathic urge urinary incontinence; } \\
\text { - Non-neurogenic urge urinary } \\
\text { incontinence. }\end{array}$ & $\begin{array}{l}\text { Patients with overactive bladder and urinary incontinence } \\
\text { with a known cause (eg, surgery, pregnancy, benign } \\
\text { prostatic hyperplasia, bladder outlet obstruction; } \\
\text { Neurogenic overactive bladder; } \\
\text { Stress urinary incontinence; } \\
\text { Bladder oversensitivity; } \\
\text { Bladder hypersensitivity; } \\
\text { Mixed populations where results not reported separately } \\
\text { for idiopathic overactive bladder subgroup. }\end{array}$ \\
\hline Intervention & $\begin{array}{l}\text { OnabotulinumtoxinA (all doses); } \\
\text { Mirabegron (all doses). }\end{array}$ & $\begin{array}{l}\text { Other variants of botulinum toxin such as Dysport } \\
\text { (abobotulinumtoxinA). }\end{array}$ \\
\hline Comparator & $\begin{array}{l}\text { OnabotulinumtoxinA; } \\
\text { Mirabegron; } \\
\text { Sacral nerve stimulation; } \\
\text { Percutaneous tibial nerve stimulation; } \\
\text { Anticholinergics (including solifenacin/ } \\
\text { tolterone); } \\
\text { Sham treatments; } \\
\text { Placebo; } \\
\text { Best supportive care. }\end{array}$ & $\begin{array}{l}\text { Other variants of botulinum toxin such as Dysport } \\
\text { (abobotulinumtoxinA). }\end{array}$ \\
\hline Outcomes & $\begin{array}{l}\text { Episodes of urinary incontinence: } \\
\text { - Change in number of episodes; } \\
\text { - Proportion of patients who achieve } \\
\geq 50 \% \text { reduction in number of episodes } \\
\text { Urinary frequency; } \\
\text { Urgency; } \\
\text { Nocturia. }\end{array}$ & \\
\hline Study design & $\begin{array}{l}\text { Randomised controlled trials; } \\
\text { Cross-over randomised controlled trials if } \\
\text { data reported at cross-over. }\end{array}$ & $\begin{array}{l}\text { Retrospective studies; } \\
\text { - Non-English reports; } \\
\text { - Abstracts/conference presentations. }\end{array}$ \\
\hline
\end{tabular}

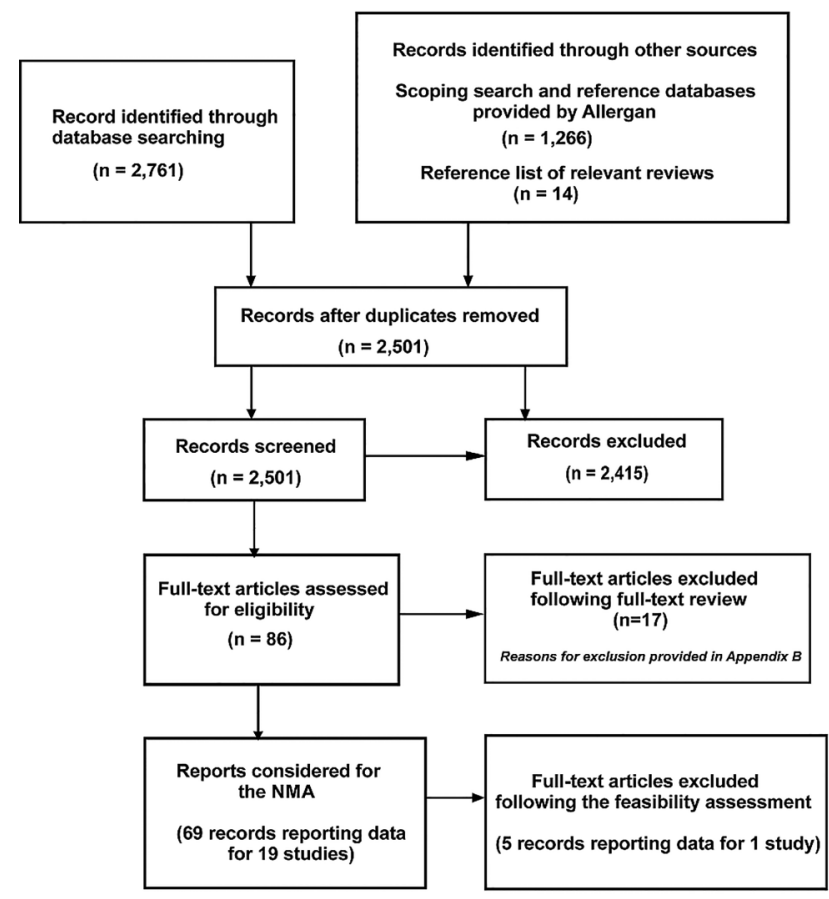

$50 \mathrm{mg}$ ), although studies evaluating any dosages were eligible to contribute to the networks where they provided links between the licensed doses through a common comparator. Networks were developed for each of the seven outcomes, based on quality of study methods, confounding factors (participants and circumstances), common treatment arms and outcomes (see online supplementary appendices C2-C7). Studies that were considered similar based on the modified PBAC tool (detailed in online supplementary appendix C1) and reported sufficient data at 12 weeks were included in the NMAs; those that neither compared key treatments nor provided indirect information did not contribute to the networks. For change from baseline outcomes, studies with no information on the mean baseline values (of the outcome) could not be included in the network metaregressions (NMRs). The outcomes of interest were: proportions of patients achieving $100 \%$ and $50 \%$ reduction from baseline in the number of UI episodes per day; mean change in UI episodes per day; proportion of patients achieving $100 \%$ reduction from baseline in the number of urgency episodes per day; mean change in urgency episodes per day; mean change in the

Figure 1 Study flow diagram. NMA, network meta-analysis. 
frequency of urination per day; mean change in the number of nocturia episodes per night.

Standard Bayesian NMA was applied to all outcomes. ${ }^{22}$ Both fixed-effects and random-effects models were conducted, but only random-effects models are presented in this publication because they account for heterogeneity in the treatment effects between studies. For some UI and urgency outcomes, differences in baseline severity between the mirabegron and onabotulinumtoxinA trials were identified. For each of these outcomes, individual patient data from two onabotulinumtoxinA trials ${ }^{23} 24$ were used to explore the relationship between baseline severity and treatment effect. Generalised linear models were used to assess whether there was a linear relationship between baseline severity and the treatment effect (OR for binomial outcomes, mean difference in change from baseline for continuous outcomes) for onabotulinumtoxinA compared with placebo. Where baseline severity had a statistically significant impact on the treatment effect, random-effects NMR was applied in addition to the NMA (further details of both the NMA and the NMR are included in online supplementary appendices D-D1). All analyses were conducted using WinBUGS V.1.4.3. ${ }^{25}$ Relative treatment effects are summarised by point estimates and $95 \%$ credible intervals (CrIs; CrIs are the Bayesian analogue of CIs; for the binomial outcomes, CrIs that exclude 1 suggest a difference between treatments; for the continuous outcomes, CrIs that exclude 0 suggest a difference between treatments). For each NMR, the relative treatment effects are reported based on the average baseline severity of the participants included in the network (ie, 3.48 episodes per day for UI and 6.32 episodes per day for urgency).

\section{RESULTS}

\section{Included studies}

The search process yielded 2501 studies of which 19 met the inclusion criteria: 11 studies assessed onabotulinumtoxinA ${ }^{232426-34}$ and 8 studies assessed mirabegron. ${ }^{35-42}$

All of the included trials were conducted postyear 2000 and none of them directly compared onabotulinumtoxinA with mirabegron. The study methods were not described in sufficient detail for 13 of the 19 trials included in the review. All but two trials ${ }^{26} 28$ were reported to be double-blind, and only six studies reported adequate concealment of randomisation. ${ }^{32-34} 364042$ The majority of studies reported a treatment period of 12 weeks, with follow-up ranging from 2 to 24 weeks or 6 months. Where reported (15 studies), loss to follow-up ranged from $0 \%^{26}{ }^{32}$ to $18.4 \%^{30}$ in studies of onabotulinumtoxinA and from $4.7 \%^{39}$ to $23.6 \%^{41}$ in studies of mirabegron. The study summary data are shown in table 2 and further details are reported within the similarity assessment in online supplementary appendices C2-C7.
Patient baseline characteristics were not reported in detail for all of the included studies. Participants' characteristics were considered within the similarity assessment (see online supplementary appendix C5). The participants were predominantly female $(55.9$ $-100 \%$ ) and had experienced $\mathrm{OAB}$ for 40.6-94 months. Patients in the mirabegron and onabotulinumtoxinA studies differed at baseline in the severity of their symptoms with a lower average number of daily UI episodes and urgency episodes observed in mirabegron studies (1-3 UI episodes and 4.5-6 urgency episodes) compared with onabotulinumtoxinA studies (5-6 UI episodes and 7-9 urgency episodes). Prior therapy for $\mathrm{OAB}$ (drugs or continence surgery) was not reported consistently across the studies.

\section{Similarity assessment}

The trials were broadly similar in terms of trial methodology, confounding factors in relation to participant populations and circumstances, and treatment arms (see online supplementary appendices C2-C7). However, some notable differences were identified that could affect potential inclusion in an indirect treatment comparison: differences in treatment period, severity of symptoms, prior therapy and treatment administration (details in online supplementary appendices C5-C7).

\section{Possible networks}

All trials that contributed data to at least one outcome are shown in the network diagram (figure 2), although the actual networks for each outcome differed depending on the data reported in each trial (summarised in the online supplementary appendices E1-E2).

\section{Assessments of heterogeneity}

Pairwise comparisons of onabotulinumtoxinA $100 \mathrm{U}$ versus placebo generally had minimal to low heterogeneity $\left(\mathrm{I}^{2}<5 \%\right)$, indicating that the results were similar across all studies. For urinary frequency, moderate heterogeneity $\left(\mathrm{I}^{2} \approx 50 \%\right)$ was observed, indicating some variation between studies above that explained by chance. The pairwise comparison of onabotulinumtoxinA 100 vs $150 \mathrm{U}$ for $100 \%$ reduction in UI episodes/day also had moderate heterogeneity $\left(\mathrm{I}^{2} \approx 60 \%\right)$. All comparisons of mirabegron versus placebo showed minimal heterogeneity (table 3 ). In addition, the networks had no potential for inconsistency between direct and indirect evidence. ${ }^{43}$

\section{Network meta-analysis}

Point estimates for fixed-effects models were consistent with those for random-effects models (see online supplementary appendix F); results of random-effects models are presented. Summary plots for onabotulinumtoxinA versus mirabegron 50 and $25 \mathrm{mg}$ are included in figures 3 and 4 (detailed forest plots are shown in online supplementary figures G1-G7), with results of the analyses summarised in table 3 . All outcomes were analysed at 12 weeks. 
Table 2 Summary of characteristics of included trials

\begin{tabular}{|c|c|c|c|c|c|}
\hline Author/trial & $\begin{array}{l}\text { Study and } \\
\text { design }\end{array}$ & $\begin{array}{l}\text { Population } \\
\text { (1) Mean age } \\
\text { (years) } \\
\text { (2) Female (\%) }\end{array}$ & $\begin{array}{l}\text { OAB inadequately } \\
\text { managed by } \\
\text { anticholinergics }\end{array}$ & $\begin{array}{l}\text { Interventions/ } \\
\text { comparators }\end{array}$ & $\begin{array}{l}\text { Duration of treatment/ } \\
\text { follow-up }\end{array}$ \\
\hline \multicolumn{6}{|l|}{ BTX studies } \\
\hline \multirow[t]{2}{*}{ Al Taweel ${ }^{26}$} & $\mathrm{RCT}$ & (1) NR & \multirow[t]{2}{*}{ Yes } & BTX: 2 doses & \multirow[t]{2}{*}{9 months } \\
\hline & $\begin{array}{l}\text { Single centre } \\
\text { (Saudi Arabia) }\end{array}$ & (2) NR & & $(200 / 100$ U) & \\
\hline \multirow[t]{2}{*}{ Brubaker $^{27}$} & Double-blind RCT & (1) $64.7-69.2$ & \multirow[t]{2}{*}{ Yes } & BTX: $200 \mathrm{U}$ & \multirow[t]{2}{*}{12 months $/ \leq 1$ month } \\
\hline & Multicentre (USA) & (2) 100.0 & & Placebo & \\
\hline \multirow[t]{2}{*}{ Chapple $^{23}$} & Double-blind RCT & (1) $59.2-59.5$ & \multirow[t]{2}{*}{ Yes } & BTX: $100 \mathrm{U}$ & \multirow{2}{*}{$\begin{array}{l}12 \text { weeks/12 weeks } \\
\text { (non-placebo-controlled) }\end{array}$} \\
\hline & $\begin{array}{l}\text { Multicentre } \\
\text { (Europe, USA) }\end{array}$ & (2) $84.5-88.1$ & & Placebo & \\
\hline \multirow{2}{*}{ Cohen $^{28}$} & $\mathrm{RCT}$ & (1) NR & \multirow[t]{2}{*}{ Yes } & BTX: 2 doses & \multirow[t]{2}{*}{24 weeks } \\
\hline & $\begin{array}{l}\text { Single centre } \\
\text { (USA) }\end{array}$ & (2) NR & & $(150 / 100 \mathrm{U})$ & \\
\hline \multirow[t]{2}{*}{ Denys $^{29}$} & Double-blind RCT & (1) 61.6 & \multirow[t]{2}{*}{ Yes } & BTX: 3 doses & \multirow[t]{2}{*}{6 months } \\
\hline & $\begin{array}{l}\text { Multicentre } \\
\text { (France) }\end{array}$ & (2) 87.9 & & $\begin{array}{l}(150 / 100 / 50 \mathrm{U}) \\
\text { Placebo }\end{array}$ & \\
\hline \multirow[t]{2}{*}{ Dmochowski ${ }^{30}$} & Double-blind RCT & (1) 58.8 & \multirow[t]{2}{*}{ Yes } & BTX: 5 doses & \multirow[t]{2}{*}{36 weeks } \\
\hline & $\begin{array}{l}\text { Multicentre } \\
\text { (Europe, USA, } \\
\text { Canada) }\end{array}$ & (2) 92.0 & & $\begin{array}{l}(300 / 200 / 150 / \\
100 / 50 \mathrm{U}) \\
\text { Placebo }\end{array}$ & \\
\hline \multirow[t]{2}{*}{ King $^{31}$} & Double-blind RCT & (1) $60.7-64.3$ & \multirow[t]{2}{*}{ Minimal response } & BTX: $200 \mathrm{U}$ & \multirow[t]{2}{*}{6 weeks/unclear } \\
\hline & $\begin{array}{l}\text { Single centre } \\
\text { (Australia) }\end{array}$ & (2) 100.0 & & Placebo & \\
\hline \multirow[t]{2}{*}{$\mathrm{Nitti}^{24}$} & Double-blind RCT & (1) $61.0-61.7$ & \multirow[t]{2}{*}{ Yes } & BTX: $100 \mathrm{U}$ & \multirow{2}{*}{$\begin{array}{l}12 \text { weeks/12 weeks } \\
\text { (non-placebo-controlled) }\end{array}$} \\
\hline & $\begin{array}{l}\text { Multicentre (USA, } \\
\text { Canada) }\end{array}$ & (2) 88.4-90.0 & & Placebo & \\
\hline \multirow[t]{2}{*}{ Sahai $^{32}$} & Double-blind RCT & (1) $49.8-50.8$ & Unclear & BTX: $200 \mathrm{U}$ & 12 weeks/12-week open \\
\hline & $\begin{array}{l}\text { Single centre } \\
(\mathrm{UK})\end{array}$ & (2) 55.9 & & Placebo & $\begin{array}{l}\text { label extension (BTX arm } \\
\text { only) }\end{array}$ \\
\hline Tincello ${ }^{33}$ & Double-blind RCT & (1) $58.2-60.7$ & Yes & BTX: $200 \mathrm{U}$ & 6 months/extension study \\
\hline & Multicentre (UK) & (2) 100.0 & & Placebo & \\
\hline Visco ${ }^{34}$ & Double-blind RCT & (1) $56.7-59.3$ & Unclear & BTX: $100 \mathrm{U}$ & 6 months $/ 6$ months (off \\
\hline & Multicentre (USA) & (2) 100.0 & & $\begin{array}{l}\text { Anticholinergic } \\
\text { protocol }\end{array}$ & treatment) \\
\hline MBG studies & & & & & \\
\hline ARIES $^{35}$ & Double-blind RCT & (1) 60.1 & NR & MBG: 2 doses & 12 weeks/30 days \\
\hline & $\begin{array}{l}\text { Multicentre (USA, } \\
\text { Canada) }\end{array}$ & (2) 74.3 & & $\begin{array}{l}(100 / 50 \mathrm{mg}) \\
\text { Placebo }\end{array}$ & \\
\hline Astellas & Double-blind RCT & (1) $54.9-56.9$ & NR & MBG: 3 doses & 12 weeks \\
\hline $178-C L-045^{36}$ & $\begin{array}{l}\text { Multicentre } \\
\text { (Japan) }\end{array}$ & (2) $80.1-85.1$ & & $\begin{array}{l}(100 / 50 / 25 \mathrm{mg}) \\
\text { Placebo }\end{array}$ & \\
\hline BLOSSOM $^{37}$ & Double-blind RCT & (1) NR & NR & MBG: 2 doses & 4 weeks/2-week placebo \\
\hline & $\begin{array}{l}\text { Multicentre } \\
\text { (international) }\end{array}$ & (2) NR & & $\begin{array}{l}\text { (150/100 mg two } \\
\text { times daily) }\end{array}$ & follow-up \\
\hline & & & & Tolterodine ER & \\
\hline & & & & $4 \mathrm{mg}$ & \\
\hline & & & & Placebo & \\
\hline CAPRICORN $^{38}$ & Double-blind RCT & (1) 59.0 & NR & MBG: 2 doses & 12 weeks/2 weeks \\
\hline & Multicentre & (2) 68.7 & & $(50 / 25 \mathrm{mg})$ & \\
\hline & $\begin{array}{l}\text { (Europe, North } \\
\text { America) }\end{array}$ & & & Placebo & \\
\hline DRAGON ${ }^{39}$ & Double-blind RCT & (1) 57.2 & NR & MBG: 4 doses & 12 weeks \\
\hline & Multicentre & (2) 89.3 & & $(200 / 100 / 50 /$ & \\
\hline & (international: 14 & & & $25 \mathrm{mg})$ & \\
\hline & countries) & & & Placebo & \\
\hline
\end{tabular}


Table 2 Continued

\begin{tabular}{|c|c|c|c|c|c|}
\hline Author/trial & $\begin{array}{l}\text { Study and } \\
\text { design }\end{array}$ & $\begin{array}{l}\text { Population } \\
\text { (1) Mean age } \\
\text { (years) } \\
\text { (2) Female (\%) }\end{array}$ & $\begin{array}{l}\text { OAB inadequately } \\
\text { managed by } \\
\text { anticholinergics }\end{array}$ & $\begin{array}{l}\text { Interventions/ } \\
\text { comparators }\end{array}$ & $\begin{array}{l}\text { Duration of treatment/ } \\
\text { follow-up }\end{array}$ \\
\hline SCORPIO $^{40}$ & $\begin{array}{l}\text { Double-blind RCT } \\
\text { Multicentre } \\
\text { (Europe, } \\
\text { Australia) }\end{array}$ & $\begin{array}{l}\text { (1) } 59.0-59.2 \\
\text { (2) } 71.6-72.9\end{array}$ & NR & $\begin{array}{l}\text { MBG: } 2 \text { doses } \\
\text { (100/50 mg) } \\
\text { Tolterodine ER } \\
4 \text { mg } \\
\text { Placebo }\end{array}$ & 12 weeks/30 days \\
\hline TAURUS $^{41}$ & $\begin{array}{l}\text { Double-blind RCT } \\
\text { Multicentre (USA, } \\
\text { Europe, Canada, } \\
\text { South Africa, NZ, } \\
\text { Australia) }\end{array}$ & $\begin{array}{l}\text { (1) } 59.2-60.1 \\
\text { (2) } 73.9-74.1\end{array}$ & NR & $\begin{array}{l}\text { MBG: } 2 \text { doses } \\
\text { (100/50 mg) } \\
\text { Tolterodine ER } \\
4 \mathrm{mg}\end{array}$ & 12 months \\
\hline Yamaguchi $^{42}$ & $\begin{array}{l}\text { Double-blind RCT } \\
\text { Multicentre } \\
\text { (Japan) }\end{array}$ & $\begin{array}{l}\text { (1) NR } \\
\text { (2) NR }\end{array}$ & NR & $\begin{array}{l}\text { MBG: } 50 \mathrm{mg} \\
\text { Placebo }\end{array}$ & 12 weeks/2 weeks \\
\hline
\end{tabular}

\section{Urinary incontinence}

$100 \%$ and $50 \%$ Reduction from baseline in the number of UI episodes/day

A logistic regression model using individual patient data from two onabotulinumtoxinA trials ${ }^{23} 24$ showed that continuous baseline UI severity did not have a statistically significant effect on the OR comparing onabotulinumtoxinA versus placebo for either $50 \%$ or $100 \%$ reduction in UI episodes/day. Hence, only standard NMA was conducted.

Across onabotulinumtoxinA trials included in the network, complete continence was achieved by 22.9 $-55 \%$ of patients receiving onabotulinumtoxinA $100 \mathrm{U}$ compared with $6.5-10.7 \%$ of the placebo group, ${ }^{23} 2429$ and the NMA suggests that onabotulinumtoxinA $100 \mathrm{U}$ is superior to placebo. Across mirabegron trials included in the network, full continence was achieved by 41.7 $-47.1 \%$ of patients given mirabegron 25 or $50 \mathrm{mg}$ vs
$36.8-40.5 \%$ of the placebo group, ${ }^{38-40}$ and there was no evidence that either of the mirabegron doses is superior to placebo. Results of comparisons versus placebo are reported in the online supplementary figure G1. These results suggest that onabotulinumtoxinA $100 \mathrm{U}$ is more efficacious than both doses of mirabegron in achieving continence. Patients receiving onabotulinumtoxinA $100 \mathrm{U}$ had 3.49 (CrI 1.97, 6.55) and 3.54 (CrI 1.93, 6.81) greater odds of achieving $100 \%$ reduction in UI episodes than those receiving mirabegron 50 and $25 \mathrm{mg}$, respectively.

Compared with placebo, higher proportions of patients achieved a $50 \%$ reduction in daily UI episodes with onabotulinumtoxinA $100 \mathrm{U} \quad(57.5-63.5 \%$ vs 28.9 $-33.2 \%),{ }^{23}{ }^{24}$ mirabegron $50 \mathrm{mg}(70.0-72.0 \%$ vs 59.2 $-60.1 \%){ }^{38} \quad 40$ and mirabegron $25 \mathrm{mg} \quad(72.8 \%$ vs $59.2 \%) .{ }^{38}$ For $50 \%$ reduction in UI episodes, the analysis suggests that onabotulinumtoxinA $100 \mathrm{U}$ and both

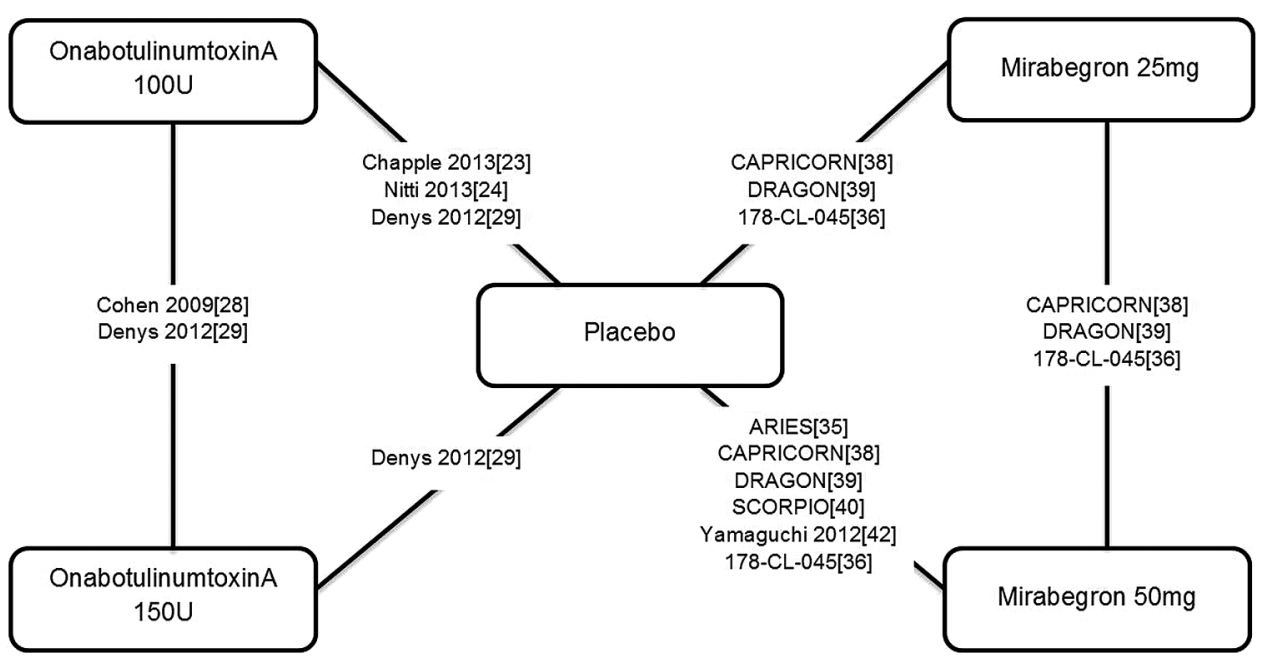

Figure 2 Network diagram showing studies contributing to the network meta-analyses. 
Table 3 Summary of outcome results for BTX (100 U) compared with MBG (50 and $25 \mathrm{mg}$ )

\begin{tabular}{|c|c|c|c|c|c|}
\hline \multirow{2}{*}{ Outcome } & \multirow[t]{2}{*}{$\begin{array}{l}\text { Heterogeneity }\left(I^{2}\right)^{*} \\
\text { (1) BTX } 100 \text { vs PBO } \\
\text { (2) MBG } 50 \text { vs PBO } \\
\text { (3) MBG } 25 \text { vs PBO }\end{array}$} & \multirow[t]{2}{*}{$\begin{array}{l}\text { Network analysis } \\
\text { (method; model; } \\
\text { prior distribution } \\
\text { for between-study } \\
\text { precision) }\end{array}$} & \multirow[t]{2}{*}{$\begin{array}{l}\text { Number of studies } \\
\text { Number of } \\
\text { patients }\end{array}$} & \multicolumn{2}{|c|}{$\begin{array}{l}\text { Summary effect } \\
\text { size (mean, } 95 \% \text { Crl) }\end{array}$} \\
\hline & & & & BTX vs MBG 50 & BTX vs MBG 25 \\
\hline \multicolumn{6}{|l|}{ Urinary incontinence } \\
\hline $\begin{array}{l}100 \% \text { reduction } \\
\text { from baseline in } \\
\text { number of } \\
\text { episodes/day }\end{array}$ & $\begin{array}{l}\text { (1) } 0 \% \\
\text { (2) } 0 \% \\
\text { (3) } 0 \%\end{array}$ & $\begin{array}{l}\text { Standard NMA; } \\
\text { random effects; } \\
\text { informative prior }\end{array}$ & $\begin{array}{l}7^{2324} 282938-40 \\
2873\end{array}$ & $\begin{array}{l}\text { OR } 3.49(1.97, \\
6.55)\end{array}$ & $\begin{array}{l}\text { OR } 3.54 \text { (1.93, } \\
6.81)\end{array}$ \\
\hline $\begin{array}{l}50 \% \text { reduction } \\
\text { from baseline in } \\
\text { number of } \\
\text { episodes/day }\end{array}$ & $\begin{array}{l}\text { (1) } 0 \% \\
\text { (2) } 0 \% \\
\text { (3) }-\dagger\end{array}$ & $\begin{array}{l}\text { Standard NMA; } \\
\text { random effects; } \\
\text { informative prior }\end{array}$ & $\begin{array}{l}4^{23243840} \\
2462\end{array}$ & $\begin{array}{l}\text { OR } 2.07(0.98, \\
4.49)\end{array}$ & $\begin{array}{l}\text { OR } 1.83(0.80, \\
4.25)\end{array}$ \\
\hline \multicolumn{6}{|l|}{ Urgency } \\
\hline $\begin{array}{l}100 \% \text { reduction } \\
\text { from baseline in } \\
\text { number of } \\
\text { episodes/day }\end{array}$ & $\begin{array}{l}(1) 0 \% \\
(2)-\dagger \\
(3)_{-}^{+}\end{array}$ & $\begin{array}{l}\text { Standard NMA; } \\
\text { random effects; } \\
\text { informative prior }\end{array}$ & $\begin{array}{l}3^{232439} \\
1961\end{array}$ & $\begin{array}{l}\text { OR } 7.01(1.62, \\
32.60)\end{array}$ & $\begin{array}{l}\text { OR } 6.16(1.43 \text {, } \\
28.58)\end{array}$ \\
\hline \multirow{3}{*}{$\begin{array}{l}\text { Change from } \\
\text { baseline in } \\
\text { number of } \\
\text { episodes/day }\end{array}$} & & & $8^{2324353638-4042}$ & Unadiusted: MD & Urinary incontinence \\
\hline & $\begin{array}{l}\text { (2) } 0 \% \\
\text { (3) } 0 \%\end{array}$ & $\begin{array}{l}\text { random effects; } \gamma \\
\text { prior }\end{array}$ & 4591 & $\begin{array}{l}-1.41(-1.84 \\
-0.98) \ddagger\end{array}$ & $\begin{array}{l}-1.32(-1.79 \\
-0.84)\end{array}$ \\
\hline & & $\begin{array}{l}\text { NMR; random } \\
\text { effects; } \gamma \text { prior }\end{array}$ & $\begin{array}{l}6 \S^{23} 2435363840 \\
3517\end{array}$ & $\begin{array}{l}\text { Adjusted: MD } \\
-0.70(-1.23 \text {, } \\
-0.16)\end{array}$ & $\begin{array}{l}\text { Adjusted: MD } \\
-0.62(-1.20 \\
-0.02)\end{array}$ \\
\hline \multicolumn{6}{|l|}{ Urgency } \\
\hline \multirow{2}{*}{$\begin{array}{l}\text { Change from } \\
\text { baseline in } \\
\text { number of } \\
\text { episodes/day }\end{array}$} & $\begin{array}{l}\text { (1) } 4.6 \% \\
\text { (2) } 0 \%\end{array}$ & $\begin{array}{l}\text { Standard NMA; } \\
\text { random effects; } \gamma\end{array}$ & $\begin{array}{l}9232429353638- \\
4042\end{array}$ & $\begin{array}{l}\text { Unadjusted: MD } \\
-1.48(-2.06,\end{array}$ & $\begin{array}{l}\text { Unadjusted: MD } \\
-1.63(-2.23,\end{array}$ \\
\hline & (3) $0 \%$ & $\begin{array}{l}\text { prior } \\
\text { NMR; } \\
\text { random-effects; } \gamma \\
\text { prior }\end{array}$ & $\begin{array}{l}6100 \\
7 \S^{23} 242935363839 \\
4390\end{array}$ & $\begin{array}{l}-0.92) \\
\text { Adjusted: MD } \\
-1.32(-2.00 \\
-0.67)\end{array}$ & $\begin{array}{l}-1.03) \\
\text { Adjusted: MD } \\
-1.49(-2.16 \\
-0.80)\end{array}$ \\
\hline \multicolumn{6}{|l|}{ Urinary frequency } \\
\hline $\begin{array}{l}\text { Change from } \\
\text { baseline in } \\
\text { number of } \\
\text { episodes/day }\end{array}$ & $\begin{array}{l}\text { (1) } 47.7 \% \\
\text { (2) } 0 \% \\
\text { (3) } 0 \%\end{array}$ & $\begin{array}{l}\text { Standard NMA; } \\
\text { random effects; } \gamma \\
\text { prior }\end{array}$ & $\begin{array}{l}8^{2324353638-4042} \\
6056\end{array}$ & $\begin{array}{l}\mathrm{MD}-0.81 \\
(-1.24,-0.40)\end{array}$ & $\begin{array}{l}M D-0.89 \\
(-1.35,-0.45)\end{array}$ \\
\hline \multicolumn{6}{|l|}{ Nocturia } \\
\hline $\begin{array}{l}\text { Change from } \\
\text { baseline in } \\
\text { number of } \\
\text { episodes/night }\end{array}$ & $\begin{array}{l}\text { (1) } 0 \% \\
\text { (2) } 0 \% \\
\text { (3) } 0 \%\end{array}$ & $\begin{array}{l}\text { Standard NMA; } \\
\text { random effects; } \gamma \\
\text { prior }\end{array}$ & $\begin{array}{l}6^{232435363942} \\
3678\end{array}$ & $\begin{array}{l}\text { MD }-0.10 \\
(-0.32,0.12)\end{array}$ & $\begin{array}{l}M D-0.06 \\
(-0.31,0.19)\end{array}$ \\
\hline $2^{2}$ values of $25 \%, 5$ & & Inaicale low, moc & 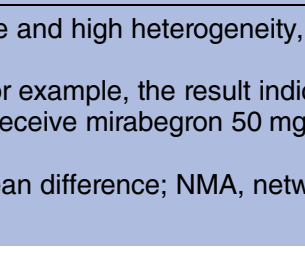 & ly. ${ }^{21}$ & eceive BTX \\
\hline
\end{tabular}

mirabegron doses are more efficacious than placebo (see online supplementary figure G2). While the NMA showed that patients receiving onabotulinumtoxinA had 2.07 times greater odds (CrI $0.98,4.49)$ than mirabegron $50 \mathrm{mg}$ and 1.83 greater odds (CrI $0.80,4.25$ ) than mirabegron $25 \mathrm{mg}$ of having $50 \%$ reduction in UI episodes, these results were inconclusive. There was only a small number of studies included in this network for this end point (four studies involving 548-773 participants). 


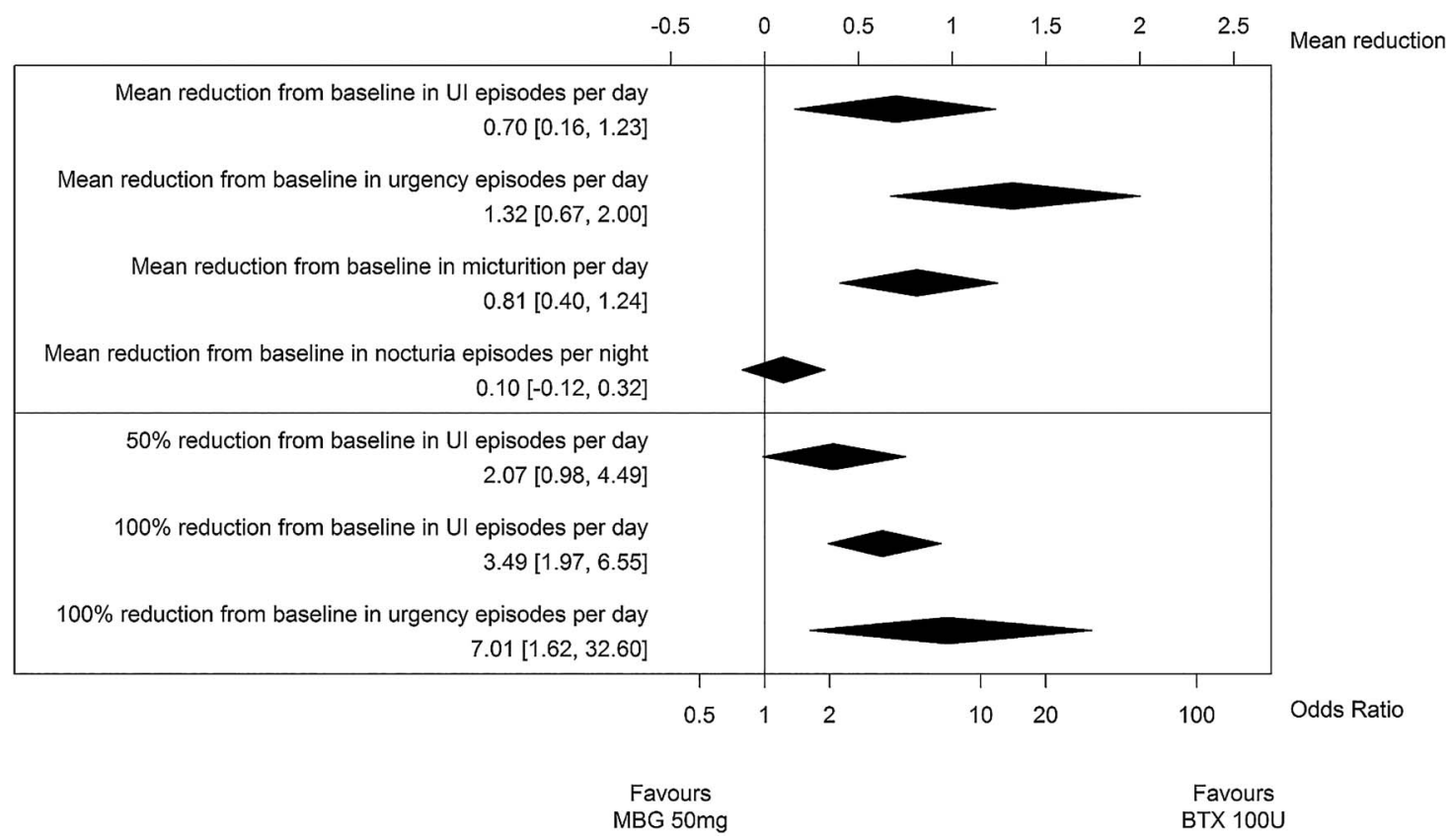

Figure 3 Summary: network meta-analysis and network metaregression for BTX $100 \mathrm{U}$ versus MBG $50 \mathrm{mg}$. BTX, onabotulinumtoxinA; MBG, mirabegron; UI, urinary incontinence.

\section{Mean reduction in UI episodes/day}

Analysis of individual patient data from two onabotulinumtoxinA trials ${ }^{23} 24$ showed that baseline number of UI episodes per day influences the mean improvement in UI episodes relative to placebo. Thus, in addition to the NMA, NMR was performed. The NMR accounts for the differences in baseline UI episodes between the studies (5-6 per day for
onabotulinumtoxinA studies vs 1-3 per day for mirabegron studies) and thus provides a fairer assessment of treatment effect.

The NMA suggests that onabotulinumtoxinA $100 \mathrm{U}$ and both mirabegron doses may be more efficacious than placebo (see online supplementary figure G.3A). In addition, onabotulinumtoxinA $100 \mathrm{U}$ may be more efficacious than both mirabegron doses (table 3).

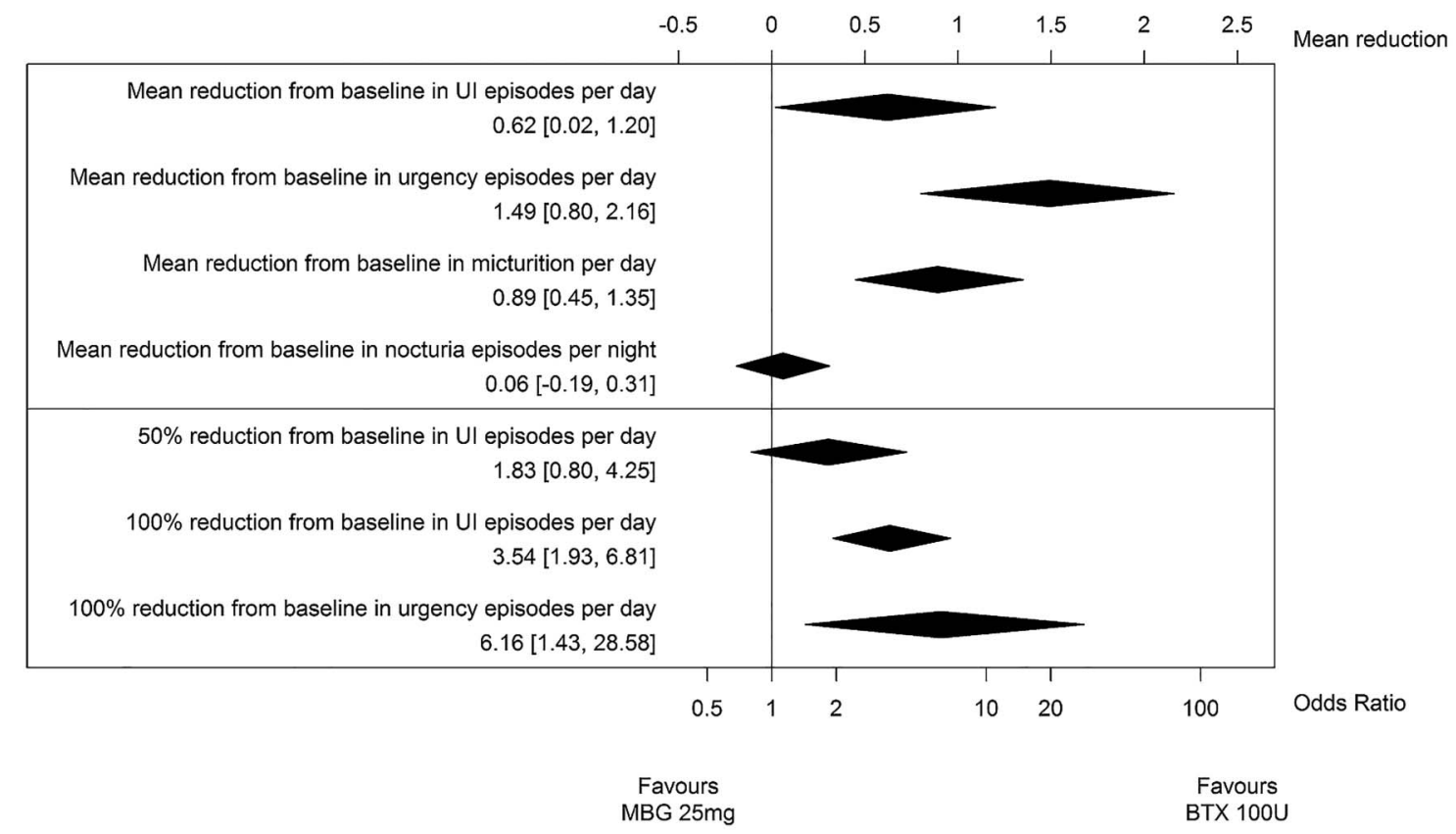

Figure 4 Summary: network meta-analysis and network metaregression for BTX $100 \mathrm{U}$ versus MBG $25 \mathrm{mg}$. BTX, onabotulinumtoxinA; MBG, mirabegron; UI, urinary incontinence. 
For the NMR, two trials did not provide any information on baseline severity and were therefore excluded. ${ }^{3942}$ Under the assumptions of the NMR (see online supplementary appendix D), the efficacy of any of the active treatments relative to placebo varies with the baseline severity of the patient, but the relative efficacy of the active treatments does not vary with the baseline severity of the patient. For patients with an average number of UI episodes at baseline (3.48 UI episodes per day across all patients included in the network), the NMR suggests that onabotulinumtoxinA $100 \mathrm{U}$ and both of the mirabegron doses are more efficacious than placebo (see online supplementary figure G3B). The average reduction relative to placebo for onabotulinumtoxinA was approximately twice that of patients receiving mirabegron, with onabotulinumtoxinA conferring on average a 108\% further improvement over mirabegron $50 \mathrm{mg}$, and $85 \%$ further improvement over mirabegron $25 \mathrm{mg}$.

On average, patients receiving onabotulinumtoxinA $100 \mathrm{U}$ improved by 0.70 episodes more per day than those receiving mirabegron $50 \mathrm{mg}$ (CrI $0.16,1.23$ ) and by 0.62 episodes more per day than those receiving mirabegron $25 \mathrm{mg}$ (CrI 0.02, 1.20). Thus, onabotulinumtoxinA $100 \mathrm{U}$ may be superior to both of the mirabegron doses.

\section{Urgency \\ $100 \%$ Reduction from baseline in the number of urgency episodes/day}

One mirabegron study ${ }^{39}$ reported the proportion of patients experiencing $100 \%$ reduction in urgency episodes (ie, no episodes at 12 weeks), and results were derived from individual patient data from two additional trials. $^{23}{ }^{24}$ Thus, three trials contributed to the network.

The NMA suggests that onabotulinumtoxinA is more efficacious than placebo for complete resolution of urgency. There was no evidence that either of the mirabegron doses is more efficacious than placebo. Results of comparisons versus placebo are reported in online supplementary figure G4. The analysis also suggests that onabotulinumtoxinA is more efficacious than mirabegron. Patients receiving onabotulinumtoxinA $100 \mathrm{U}$ had higher odds of resolving urgency episodes than those receiving mirabegron $50 \mathrm{mg}$ (7.01 times higher; CrI $1.62,32.60)$ and mirabegron $25 \mathrm{mg}$ (6.16 times higher; CrI 1.43, 28.58).

\section{Mean reduction in urgency episodes per day}

Based on the number of urgency episodes per day at baseline, patients in the onabotulinumtoxinA studies were more severe than patients in the mirabegron studies (baseline urgency episodes: 7-9 per day for onabotulinumtoxinA studies vs 4-6 per day for mirabegron studies). An analysis of individual patient data from two onabotulinumtoxinA trials ${ }^{23}{ }^{24}$ did not find a statistically significant relationship between baseline urgency and treatment effect. However, an NMR was performed, in addition to an NMA, to ensure that the differences in baseline severity did not affect the results.

The results of the NMA and NMR are similar (note that two trials did not provide any information on baseline severity and were therefore excluded from the $\mathrm{NMR}^{40}{ }^{42}$ ). The NMA suggests that all of the active treatments are more efficacious than placebo (see online supplementary figure G5A). Based on the average severity at baseline across all patients included in the network, the NMR supports this conclusion (see online supplementary figure G5B). The average reduction relative to placebo for onabotulinumtoxinA is at least three times that of patients receiving mirabegron after adjusting for baseline severity of urgency symptoms, with onabotulinumtoxinA conferring, on average, approximately three times the reduction due to mirabegron $50 \mathrm{mg}$ and four times the reduction due to mirabegron $25 \mathrm{mg}$. Both analyses suggest that onabotulinumtoxinA $100 \mathrm{U}$ is more efficacious than either mirabegron 50 or $25 \mathrm{mg}$ in reducing urgency episodes. The NMR suggests that patients improved, on average, by 1.32 (CrI 0.67, 2.00) and 1.49 (CrI 0.80, 2.16) episodes more per day on onabotulinumtoxinA $100 \mathrm{U}$ compared with mirabegron 50 and $25 \mathrm{mg}$, respectively.

\section{Urinary frequency}

At baseline, severity of urinary frequency was similar across patients in the onabotulinumtoxinA and mirabegron trials. NMA suggests that each of the active treatments is more efficacious than placebo (see online supplementary figure G6). The analysis also suggests that onabotulinumtoxinA is more efficacious than both mirabegron doses in reducing the mean number of urination episodes per day. On average, patients receiving onabotulinumtoxinA $100 \mathrm{U}$ improved by 0.81 episodes more than patients on mirabegron $50 \mathrm{mg}$ (CrI $0.40,1.24)$ and by 0.89 episodes more than those on mirabegron $25 \mathrm{mg}(\mathrm{CrI} 0.45,1.35)$.

\section{Nocturia}

Baseline severity was similar across the onabotulinumtoxinA and mirabegron trials. Each of the active treatments was shown to be more efficacious than placebo, with the mean reduction in nocturia episodes per night, relative to placebo, greater for patients receiving onabotulinumtoxinA $100 \mathrm{U} \quad(0.25$; CrI $0.07,0.43)$ than for patients receiving mirabegron $50 \mathrm{mg}(0.15$; CrI $0.03,0.27)$ and mirabegron $25 \mathrm{mg}(0.19$; CrI $0.02,0.36$; see online supplementary figure G7). However, while estimates of the mean treatment effects favoured onabotulinumtoxinA, there was no conclusive evidence of a difference between onabotulinumtoxinA $100 \mathrm{U}$ and either mirabegron $50 \mathrm{mg}$ (mean reduction 0.10; CrI $-0.12,0.32$ ) or mirabegron $25 \mathrm{mg}$ (mean reduction 0.06 ; $\mathrm{CrI}-0.19,0.31$ ) for the nocturia end point. 
DISCUSSION

Until 2012, patients being treated for OAB progressed along a clinical pathway of first-line conservative therapy which included lifestyle interventions and behavioural techniques, followed by second-line treatment with cycling on anticholinergics, then third-line treatments such as sacral neuromodulation, percutaneous tibial nerve stimulation, and off-label use of onabotulinumtoxinA. With regard to anticholinergics, there is known efficacy and safety but no clear evidence of superiority of one agent over another. In addition, long-term adherence is a problem secondary to adverse effects and insufficient efficacy. ${ }^{44}$ The recent approval of two new treatments-mirabegron and onabotulinumtoxinA-are viable alternatives to trying multiple anticholinergics, and they also allow for potential improved management of OAB symptoms. Pivotal trials of both treatments, all sponsored by their manufacturers, have shown their superiority over placebo. ${ }^{232430353840}$

Using NMA, the current review combined all relevant evidence available, on the treatment of adults with idiopathic OAB with licensed doses of onabotulinumtoxinA and mirabegron. In the absence of direct comparative evidence, seven efficacy outcomes were addressed in the indirect treatment comparison, each outcome including between three and nine studies. Although only the results of random-effects models are reported, fixed-effects models were also explored. Point estimates were consistent but the degree of uncertainty around the estimates was somewhat greater for the random-effects models, indicating some potential for heterogeneity among the studies (see online supplementary appendix F). Results from the network analyses suggest that onabotulinumtoxin $100 \mathrm{U}$ is more efficacious than mirabegron (50 and $25 \mathrm{mg}$ doses) in improving the following $\mathrm{OAB}$ parameters: UI episodes per day, $100 \%$ reduction in UI, urgency episodes per day, $100 \%$ reduction in urgency and urinary frequency. Studies included in the analyses typically assessed shortterm outcomes (12-13 weeks) as dictated by regulatory agencies; longer term efficacy of onabotulinumtoxinA versus mirabegron cannot be extrapolated from the data reported.

NMA involves pooling of trials and is subject to some of the same limitations as pairwise meta-analysis, such as quality of the individual studies and publication bias. A key consideration of any NMA is whether the identified studies are sufficiently similar to facilitate reliable comparison on an outcome of interest. The onabotulinumtoxinA and mirabegron trials were compared in terms of quality of study methods, patient populations, treatments and outcome measures. While the trials were broadly similar in terms of key study characteristics, there were notable differences. For example, the methodology of each individual trial could not be fully assessed for risk of bias given the limited information available, the majority of trials had been sponsored by the manufacturers, and not all studies reported the same degree of information. The quality and methods of the randomised trials were therefore assessed using the PBAC tool, which considers adequacy of allocation concealment, reported blinding, duration of study and loss to follow-up (summarised in the online supplementary appendix C2) . Having only a few studies in the final networks meant that for some outcomes the analysis had limited power to detect a difference between the two active treatments, as reflected in the wide CrIs on some comparative treatment effects.

There were differences in the proportion of females in the onabotulinumtoxinA studies (55.4-100\%) compared with the mirabegron studies (68.7-89.3\%), with all but one of the onabotulinumtoxinA studies involving a larger percentage of women. This NMA has made the assumption that sex does not affect response to treatment. Patients in the onabotulinumtoxinA trials had more severe UI and urgency symptoms at baseline than those in the mirabegron trials and all patients in the onabotulinumtoxinA trials had failed antimuscarinic therapy. Our logistic regression model assessed the impact of continuous baseline UI severity on OR (onabotulinumtoxinA vs placebo) and suggested that continuous baseline UI severity did not significantly impact the OR for either $50 \%$ or $100 \%$ reduction in UI episodes/day. However, a recent analysis of the effect of categorical baseline UI severity on $100 \%$ reduction in UI episodes/day did show a trend for increasing OR (onabotulinumtoxinA vs placebo) with increasing categorical baseline UI severity. ${ }^{45}$ The NMR model that we used adjusts for the baseline severity by assuming a linear relationship between baseline severity and change from baseline. This assumption was tested using individual patient data from two onabotulinumtoxinA trials. The assumption held up in this test but should still be considered a limitation of the analysis given that such patientlevel data were not available for mirabegron trials. Based on the data available, this approach was the most pragmatic approach possible. The treatments themselves also differ in their mode of administration: onabotulinumtoxinA and the placebo in the onabotulinumtoxinA trials were administered as a single procedure, while both mirabegron and placebo were administered as daily oral tablets in the mirabegron trials. In order to produce a connected network for the purposes of the analyses it was assumed that the placebo treatments would be equally effective when administered to the same populations. ${ }^{46}$ We recognise that this assumption is potentially a source of bias in the NMA.

The NMA was informed by extensive searches conducted across a range of information sources. Since only English language studies were specified in the protocol, studies published in other languages were not included in this review. The review may also be subject to publication bias; the potential impact of this was not explored further. 
Both onabotulinumtoxinA and mirabegron have side effects associated with treatment, albeit the adverse effect profiles are different from those of antimuscarinic agents. ${ }^{44}$ Most common side effects with mirabegron include hypertension, nasopharyngitis, urinary tract infection and headache, while onabotulinumtoxinA is associated with urinary tract infections, dysuria, urinary retention, bacteriuria, and increased residual urine volume. Currently, there is little evidence comparing the safety of mirabegron and onabotulinumtoxinA with other interventions for OAB. The current analysis did not address adverse events with either compound, which represents a limitation of the review.

There is less literature relating to the treatment of $\mathrm{OAB}$ with approved doses of onabotulinumtoxinA and mirabegron, probably because they are newer treatments. A Cochrane review that aimed to establish the comparative effectiveness of intravesical botulinum toxin with other treatments for neurogenic and idiopathic OAB identified only one study which compared onabotulinumtoxinA with another active treatment (intravesical resinferatoxin) ${ }^{47}$ Two meta-analyses, which combined results from the various phase III trials of mirabegron, ${ }^{48}{ }^{49}$ reported the short-term effectiveness of mirabegron $50 \mathrm{mg}$ compared with placebo, and a recent mixed treatment comparison of medical treatments for OAB identified six studies of mirabegron $50 \mathrm{mg} .{ }^{17}$

This research adds information to the current evidence base and, to the best of our knowledge, represents the first evaluation comparing the relative benefits of onabotulinumtoxinA and mirabegron. This study will hopefully assist patients and healthcare providers in making more informed decisions with regard to $\mathrm{OAB}$ treatment options. Future research building on these existing networks with other treatments for OAB, appropriate to the severity of the patients' $\mathrm{OAB}$ symptoms and corresponding phase of treatment, will continue to aid in the treatment selection process.

\section{CONCLUSIONS}

OnabotulinumtoxinA and mirabegron have recently received marketing authorisations for patients with $\mathrm{OAB}$ and offer potential alternatives to management with anticholinergic therapy in patients with inadequately managed symptoms. In this indirect treatment comparison, baseline differences between the onabotulinumtoxinA and mirabegron studies were adjusted using NMR. Results of this NMR suggest that, compared with mirabegron, onabotulinumtoxinA is associated with additional reductions in the number of UI episodes and urgency episodes per day and daily urinary frequency in patients with idiopathic $\mathrm{OAB}$ at 12 weeks. In addition, more patients receiving onabotulinumtoxinA experience a $100 \%$ reduction in UI and $100 \%$ reduction in urgency than patients receiving mirabegron.

OnabotulinumtoxinA is a promising therapeutic alternative for patients that have failed anticholinergic treatment. There is a need for more supporting evidence from well-conducted, head-to-head comparisons of onabotulinumtoxinA and other therapies evaluating outcomes and adverse effects over both the short and long term.

Author affiliations

${ }^{1}$ Department of Primary Care and Population Health, University College London, London, UK

${ }^{2}$ USC Institute of Urology, Keck School of Medicine of USC, Los Angeles, California, USA

${ }^{3}$ York Health Economics Consortium, University of York, York, UK

${ }^{4}$ Quantics, Edinburgh, UK

${ }^{5}$ Allergan, Inc., Irvine, California, USA

${ }^{6}$ Xcenda, Palm Harbor, Florida, USA

${ }^{7}$ Allergan Holdings Ltd, Marlow, UK

${ }^{8}$ Allergan, Inc., Bridgewater, New Jersey, USA

${ }^{9}$ Celgene, Summit, New Jersey, USA

Acknowledgements The authors wish to acknowledge Dr Sam Brown (YHEC), Ms Danielle Varley (YHEC), Andrew Messali (Allergan) and Rick Morton (Allergan) for additional project assistance and input, Dr Ann Yellowlees (Quantics) for contributions to the statistical analysis and review of the manuscript, Zsolt Hepp (Allergan) for assistance with editing of the manuscript, and Dr Maria G Cikalo for medical writing assistance with the manuscript.

Contributors $\mathrm{KK}, \mathrm{CL}, \mathrm{JG}$ and $\mathrm{RM}$ were involved in the inception and design of the study. MA performed the literature searches. MA, RM and Sam Brown were involved in study identification and data acquisition. RM, KF and QN performed the statistical analysis. KK, CL, JG, RM, MA, KF, QN, NF and DAG critically reviewed the manuscript for intellectual content and revised the draft.

Funding This research and subsequent manuscript generation was funded by Allergan.

Competing interests NF has received funding for research and consulting from Allergan and Ipsen Pharma, both of whom manufacture Botulinum toxin for various indications. DAG is a consultant for Allergan and investigator for Astellas. YHEC (RM, MA, JG) and Quantics (KF) received funding to carry out the review and indirect treatment comparison from Allergan. $C L$ is an employee of Allergan Holdings Ltd. QN and KK have no competing interests to declare.

Provenance and peer review Not commissioned; externally peer reviewed.

Data sharing statement Additional supplementary methodology and data for the analysis are available online. The search strategies for data sources other than MEDLINE and Embase are available on request.

Open Access This is an Open Access article distributed in accordance with the Creative Commons Attribution Non Commercial (CC BY-NC 4.0) license, which permits others to distribute, remix, adapt, build upon this work noncommercially, and license their derivative works on different terms, provided the original work is properly cited and the use is non-commercial. See: http:// creativecommons.org/licenses/by-nc/4.0/

\section{REFERENCES}

1. Abrams P, Artibani W, Cardozo L, et al. Reviewing the ICS 2002 terminology report: the ongoing debate. Neurourol Urodyn 2009;28:287.

2. Brown JS, McGhan WF, Chokroverty S. Comorbidities associated with overactive bladder. Am J Manag Care 2000;6:S574-9.

3. Rackley RR, Vasavada SP, Firoozi F, et al. Neurogenic bladder. Medscape, 2009. http://emedicine.medscape.com/article/ 453539-overview\#a11 (accessed Aug 2014).

4. Liberman JN, Hunt TL, Stewart WF, et al. Health-related quality of life among adults with symptoms of overactive bladder: results from a U.S. community-based survey. Urology 2001;57:1044-50.

5. Tromp AM, Smit JH, Deeg DJ, et al. Predictors for falls and fractures in the longitudinal aging study Amsterdam. J Bone Miner Res 1998;13:1932-9. 
6. Thüroff JW, Abrams $\mathrm{P}$, Andersson $\mathrm{KE}$, et al. EAU guidelines on urinary incontinence. Eur Urol 2011;59:387-400.

7. Gormley EA, Lightner DJ, Burgio KL, et al. Diagnosis and treatment of overactive bladder (non-neurogenic) in adults: AUA/SUFU guideline. J Urol 2012;188(6 Suppl):2455-63.

8. Leong RK, De Wachter SG, van Kerrebroeck PE. Current information on sacral neuromodulation and botulinum toxin treatment for refractory idiopathic overactive bladder syndrome: a review. Urol Int 2010;84:245-53.

9. National Institute for Health and Care Excellence (NICE). NICE clinical guideline 171. Urinary incontinence: the management of urinary incontinence in women. London: NICE, 2013. http://www.nice.org.uk/ guidance/cg171/chapter/recommendations (accessed Aug 2014).

10. Chancellor MB, Levanovich P, Rajaganapathy BR, et al. Optimum management of overactive bladder: Medication vs Botox $®$ vs InterStim $\AA$ vs Urgent $₫$ PC. Urol Pract 2014;1:7-12.

11. Wang $\mathrm{Y}, \mathrm{Xu} \mathrm{K}, \mathrm{Hu} \mathrm{H}$, et al. Prevalence, risk factors, and impact on health related quality of life of overactive bladder in China. Neurourol Urodyn 2011;30:1448-55.

12. Abrams P, Manson J, Kirby MG. Incidence and epidemiology of storage lower urinary tract symptoms. Eur Urol Rev 2012;7:50-4.

13. BOTOX. Approved label. Suppl. 5251 (01/18/2013). http://www. accessdata.fda.gov (accessed Aug 2014)

14. Summary of Product Characteristics: BOTOX, Allergan Ltd. May 2014. http://www.medicines.ie (accessed Aug 2014).

15. Summary of Product Characteristics: BOTOX, Allergan Ltd. January 2014. http://www.mhra.gov.uk (accessed Aug 2014).

16. Betmiga: EPAR-Product Information. http://www.ema.europa.eu (accessed Aug 2014).

17. Maman K, Aballea S, Nazir J, et al. Comparative efficacy and safety of medical treatments for the management of overactive bladder: a systematic literature review and mixed treatment comparison. Eur Urol 2014;65:755-65.

18. Papanicolaou P, McKendrick J, Deem M, et al. Systematic literature review of the burden of (idiopathic) overactive bladder. Fleet, Hampshire: PRMA Consulting, 2013.

19. Salanti G. Indirect and mixed-treatment comparison, network, or multiple-treatments meta-analysis: many names, many benefits, many concerns for the next generation evidence synthesis tool. Res Synth Methods 2012;3:80-97.

20. Australian Pharmaceutical Benefits Advisory Committee (PBAC) Indirect Comparisons Working Group. Report of the indirect comparisons working group to the pharmaceutical benefits advisory committee: assessing indirect comparisons. Canberra: Australian Department of Health and Ageing, PBAC, 2009.

21. Higgins JP, Thompson SG, Deeks JJ, et al. Measuring inconsistency in meta-analyses. BMJ 2003;327:557-60.

22. Dias S, Welton NJ, Sutton AJ, et al. NICE DSU technical support document 2: a generalised linear modelling framework for pairwise and network meta-analysis of randomised controlled trials. Sheffield: Decision Support Unit, 2011.

23. Chapple C, Sievert KD, MacDiarmid S, et al. OnabotulinumtoxinA $100 \mathrm{U}$ significantly improves all idiopathic overactive bladder symptoms and quality of life in patients with overactive bladder and urinary incontinence: a randomised, double-blind, placebo-controlled trial. Eur Urol 2013;64:249-56.

24. Nitti VW, Dmochowski R, Herschorn S, et al. OnabotulinumtoxinA for the treatment of patients with overactive bladder and urinary incontinence: results of a phase 3 , randomized, placebo controlled trial. J Urol 2013;189:2186-93.

25. Lunn DJ, Thomas A, Best N, et al. WinBUGS-a Bayesian modelling framework: concepts, structure, and extensibility. Stat Comput 2000;10:325-37.

26. Altaweel W, Mokhtar A, Rabah DM. Prospective randomized trial of $100 u$ vs $200 u$ BOTOX $\circledast$ in the treatment of idiopathic overactive bladder. Urol Ann 2011;3:66-70.

27. Brubaker L, Richter HE, Visco A, et al. Refractory idiopathic urge urinary incontinence and botulinum A injection. J Urol 2008;180:217-22.

28. Cohen BL, Barboglio $\mathrm{P}$, Rodriguez $\mathrm{D}$, et al. Preliminary results of a dose-finding study for botulinum toxin-a in patients with idiopathic overactive bladder: 100 versus 150 units. Neurourol Urodyn 2009;28:205-8.

29. Denys P, Le Normand L, Ghout I, et al. Efficacy and safety of low doses of onabotulinumtoxinA for the treatment of refractory idiopathic overactive bladder: a multicentre, double-blind, randomised, placebo-controlled dose-ranging study. Eur Urol 2012;61:520-9.
30. Dmochowski R, Chapple C, Nitti VW, et al. Efficacy and safety of onabotulinumtoxina for idiopathic overactive bladder: a double-blind, placebo controlled, randomized, dose ranging trial. $J$ Urol 2010;184:2416-22.

31. King J, Neville J. A randomised, double-blind, placebo-controlled trial of botulinum toxin type $A$ injections for the treatment of refractory idiopathic detrusor overactivity[abstract]. Int Urogynecol J Pelvic Floor Dysfunct 2007;18(Suppl 1):S77.

32. Sahai A, Khan MS, Dasgupta P. Efficacy of botulinum toxin-A for treating idiopathic detrusor overactivity: results from a single center, randomized, double-blind, placebo controlled trial. J Urol 2007;177:2231-6.

33. Tincello DG, Kenyon S, Abrams KR, et al. Botulinum toxin a versus placebo for refractory detrusor overactivity in women: a randomised blinded placebo-controlled trial of 240 women (the RELAX study). Eur Urol 2012;62:507-14.

34. Visco AG, Brubaker L, Richter HE, et al. Anticholinergic therapy vs. onabotulinumtoxinA for urgency urinary incontinence. N Engl J Med 2012;367:1803-13.

35. Nitti VW, Auerbach S, Martin N, et al. Results of a randomized phase III trial of mirabegron in patients with overactive bladder. J Urol 2013;189:1388-95

36. Astellas. A Phase 2, double blind, placebo controlled, parallel group study of YM 178 in patients with overactive bladder (178-CL-045). Clinical Study Report Synopsis. Bethesda, MD: Astellas, 2009.

37. Astellas. A randomized, double blind, parallel group, proof-of-concept study of YM178 in comparison with placebo and tolterodine in patients with symptomatic overactive bladder (BLOSSOM) 178-CL-008. Clinical Study Report Synopsis. Bethesda, MD: Astellas, 2006.

38. Herschorn S, Barkin J, Castro-Diaz D, et al. A phase III, randomized, double-blind, parallel-group, placebo-controlled, multicentre study to assess the efficacy and safety of the $\beta_{3}$ adrenoceptor agonist, mirabegron, in patients with symptoms of overactive bladder. Urology 2013;82:313-20.

39. Chapple CR, Dvorak V, Radziszewski P, et al. A phase II dose-ranging study of mirabegron in patients with overactive bladder. Int Urogynecol J 2013;24:1447-58.

40. Khullar V, Amarenco G, Angulo JC, et al. Efficacy and tolerability of mirabegron, a $\beta(3)$-adrenoceptor agonist, in patients with overactive bladder: results from a randomised European-Australian phase 3 trial. Eur Urol 2013;63:283-95.

41. Chapple CR, Kaplan SA, Mitcheson D, et al. Randomized double-blind, active-controlled phase 3 study to assess 12-month safety and efficacy of mirabegron, a (3)-adrenoceptor agonist, in overactive bladder. Eur Urol 2013;63:296-305.

42. Yamaguchi $\mathrm{O}$, Hidehiro $\mathrm{K}$, Yukio $\mathrm{H}$, et al. A phase III, randomized, double-blind, placebo and active controlled study of once-daily mirabegron $50 \mathrm{mg}$ in patients with overactive bladder[abstract]. Urology 2012;80(Suppl 1):S10.

43. Dias S, Welton NJ, Sutton AJ, et al. NICE DSU technical support document 4: inconsistency in networks of evidence based on randomised controlled trials. London: National Institute of Health and Care Excellence, 2011

44. Andersson KE. New developments in the management of overactive bladder: focus on mirabegron and onabotulinumtoxin A. Ther Clin Risk Manag 2013;9:161-70.

45. Drake M, Ginsberg D, Gruenenfelder J, et al. Onabotulinumtoxin A improves the symptoms of urgency and incontinence and provides treatment benefit in patients with overactive bladder regardless of incontinence severity at baseline. Eur Urol Supp/ 2015;2:e148.

46. Salanti G. Indirect and mixed-treatment comparison, network, or multiple-treatments meta-analysis: many names, many benefits, many concerns for the next generation evidence synthesis tool. Res Syn Meth 2012;3:80-97.

47. Duthie JB, Vincent M, Herbison GP, et al. Botulinum toxin injections for adults with overactive bladder syndrome. Cochrane Database Syst Rev 2011;(12):CD005493.

48. Nitti VW, Khullar V, van Kerrebroeck P, et al. Mirabegron for the treatment of overactive bladder: a prespecified pooled efficacy analysis and pooled safety analysis of three randomised, double-blind placebo-controlled, phase III studies. Int J Clin Pract 2013;67:619-32.

49. Cui $\mathrm{Y}$, Zong $\mathrm{H}$, Yang $\mathrm{C}$, et al. The efficacy and safety of mirabegron in treating OAB: a systematic review and meta-analysis of phase III trials. Int Urol Nephrol 2014;46:275-84. 